\title{
Bone and brain metastases of endometrial carcinoma and its treatment with radiotherapy
}

Keywords: cancer, pelvic recurrence, lymph nodes, liver, adrenals, brain, bones, soft tissue

\section{Introduction}

Cancer of the uterine body is the fourth in worldwide incidence in women. ${ }^{1}$ In developed countries, endometrial cancer is the fourth most common cancer in women. ${ }^{2}$ Typical sites of recurrent and endometrial carcinoma include local pelvic recurrence, pelvic and para-aortic nodes, peritoneum and lungs. Atypical sites include extra-abdominal lymph nodes, liver, adrenals, brain, bones and soft tissue. ${ }^{3}$ Bone metastases with endometrial cancer are infrequent. ${ }^{4}$ Female genital tract cancers, are considered "neurophobic" since brain metastases from female genital tract cancers, apart from choriocarcinoma, are rare and usually develop as part of a widespread disseminated disease. ${ }^{5}$

\section{Case report}

A 55year-old woman, with one previous gestation and a cesarean section, without other antecedents of importance. Began her current illness with abnormal vaginal bleeding in January and July 2015. She had curettage and biopsy on July 30, 2015, with a histopathological result of $2^{\text {nd }}$ grade endometrial adenocarcinoma. She was treated with histerectomy, bilateral ovarian resection and biopsies of the bladder, omentum and vagina, on August $20^{\text {th }}, 2015$, with a pathology result: moderately differentiated endometrioid adenocarcinoma of low grade according to binary classification, Invading the entire wall without reaching the serosa, with vascular and lymphatic invasion; Left parametrium with metastases, fragments of bladder with metastases, the rest without invasion.

Radiotherapy complete pelvic cycle, 50 Gy in 25 sessions with linear accelerator from September 28 to November 3, 2015. High dose brachytherapy 30Gy in 4 sessions, in November 5, 9, 12 and 17 , separated by half a centimeter of the vaginal mucosa. 6 cycles of chemotherapy with carboplatin and paclitaxel. 3 cycles with cisplatin and doxorubicin. PET-CT of February 2017 with an increase in size and number of cervical, mediastinal, retroperitoneal, axillary and pelvic nodes; new lesions in bone, liver, lung; with increased uptake. Data on venous thrombosis in the pelvic limb. Lumbar thoracic osteoarthrosis, renal cyst. She received tamoxifen 5 months. Presented right pelvic edema. Received 30 Gy radiotherapy in 10 sessions to the lumbar area and right pelvic inguinal zone in March 2-15,2017, evolving with decreased pain.

She started monthly zoledronic acid; and also 3 cycles of Carboplatin AUC5, Paclitaxel 175mg/m2 until May 2017, in which a CT scan of the skull, neck, thorax, abdomen and pelvis was found with: lesion in the left cerebral capsule of $19 \mathrm{~mm}$, left cervical adenopathy in the VA level of $30 \mathrm{~mm}$, pulmonary lesion of $16 \mathrm{~mm}$, lesion adjacent to the right internal iliac vein of $19 \mathrm{~mm}$, and result of us venous doppler with venous thrombosis at the level of common and superficial femoral and poplitea. The patient was sent to radiation
Volume 3 Issue 4 - 2017

Nora Hilda Chávez Hernández
Instituto Mexicano del Seguro Social, México

Correspondence: Nora Hilda Chávez Hernández, Unidad Médica de Atención Ambulatoria 180, Instituto Mexicano del Seguro Social,Valle del Chalco (Estado de México), México, Email norahildachavez@hotmail.com

Received: June 01, 2017| Published: July 10, 2017

oncology and angiology (Figure 1-3).

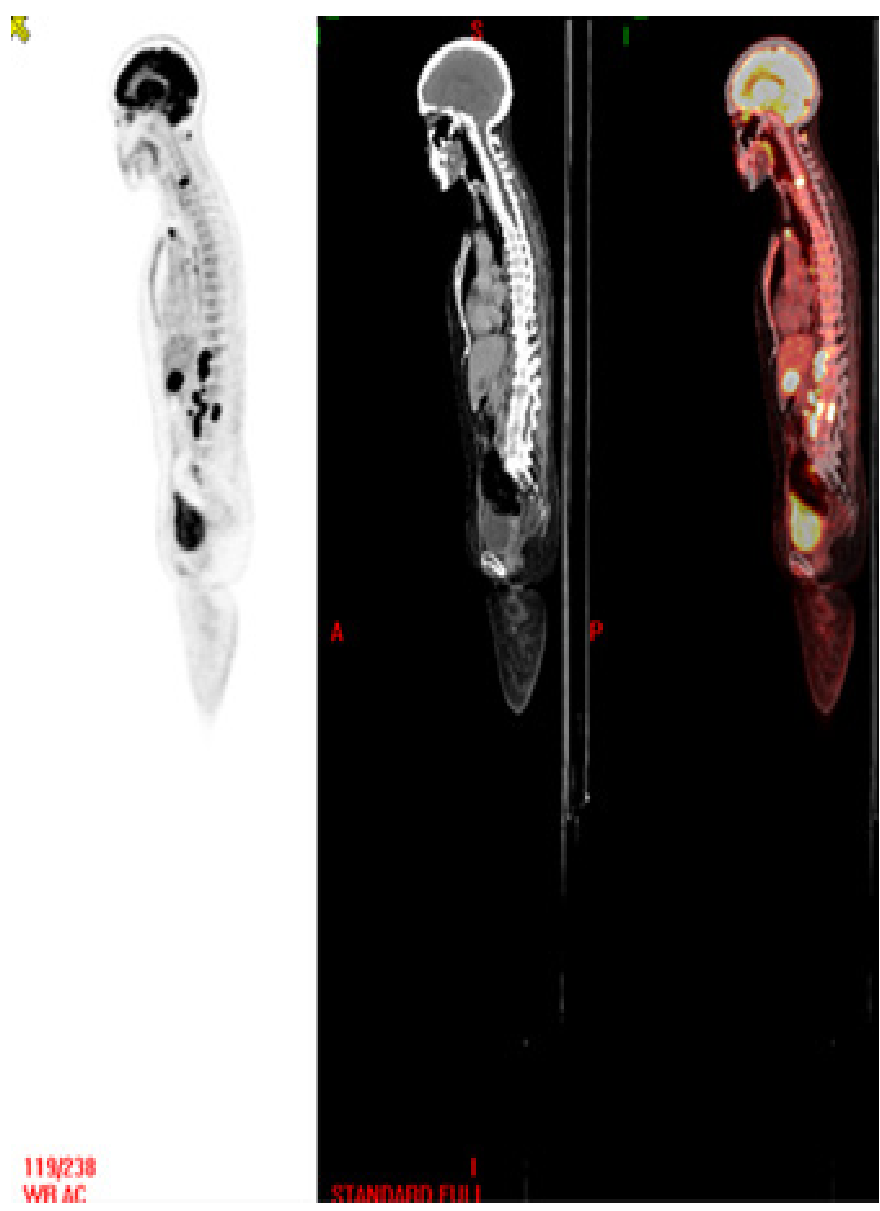

Figure I Sagital positron emission tomography. 


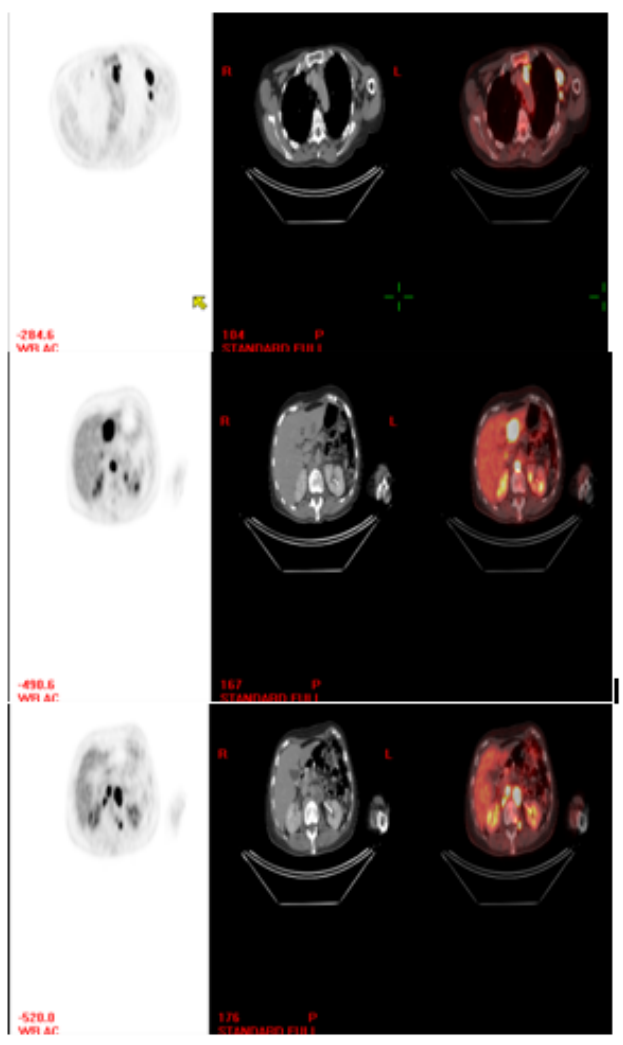

Figure 2 Axial PET-CT.

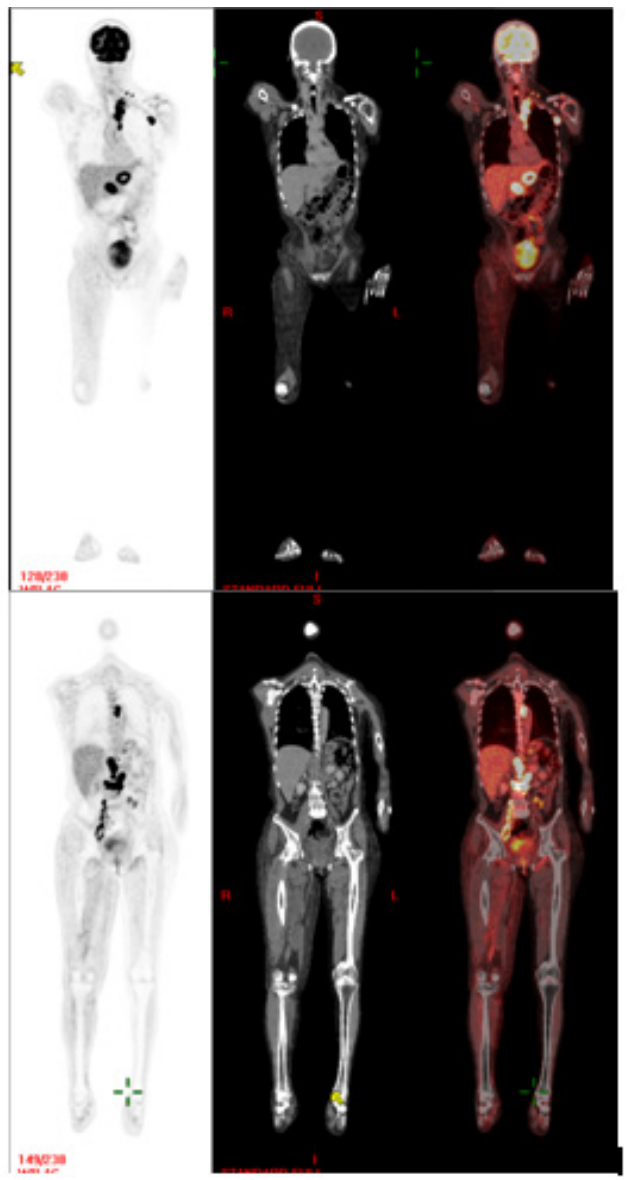

Figure 3 Coronal. PET-CT.

\section{Discussion}

According to the literature, the patient had bone and cerebral metastases when the disease was very disseminated. ${ }^{5}$ Radiotherapy is a successful and time efficient method by wich to palliate pain and/or prevent the morbidity of bone metastases. External beam radiotherapy has been the mainstay for the treatment of pain and/or prevention of the morbidity caused by bone metastases. Various fractionation schedules can provide significant palliation of symptoms and/or prevent the morbidity of bone metastases. ${ }^{6}$

Radiotherapy is capable of improving stability of osteolytic spinal metastases from gynecologic cancer by facilitating re-ossification. Patients who received chemotherapy prior to radiotherapy may require additional bone supportive treatment to overcome bone remodeling imbalance. Survival in women with bone metastases from gynecologic cancer remains por. ${ }^{7}$ Radiation therapy has provided of good results in more than half of the patients with bone metastases, with relief of pain and manteinance of quality of life in previous reports. ${ }^{8}$

The radiation dose to the osseous metastasis that the patient received had previously been proven effective in a RTOG study. ${ }^{9}$ The median survival after diagnosis of brain metastases from endometrial carcinoma was 5months; however, a better survival has been achieved with multimodal therapy including surgical resection or stereotactic radiosurgery followed by whole brain radiotherapy and/or chemotherapy. The patient has been sent with the radiation oncologist.

\section{Acknowledgements}

None.

\section{Conflict of interest}

Author declares that there is no conflict of interest.

\section{References}

1. Ferlay J, Soerjomataram I, Ervik M, et al. Globocan 2012: Estimated Cancer Incidence, Mortality and Worldwide in 2012. World Health Organization; 2013.

2. Colombo N, Preti E, Landoni F, et al. Endometrial cáncer: ESMO Clinical Practice Guidelines for diagnosis, treatment and follow -up. Ann Oncol. 2013;24(6):vi35-vi39.

3. Kurra V, Krajewski KM, Jagannathan J, et al. Typical and atypical metastastatic sites of recurrent endometrial carcinoma. Cancer Imaging. 2013;13(1):113-122.

4. Uccella S, Morris JM, Bakkum-Gamez JN, et al. Bone metastases in endometrial cancer: Report on 19 patients and review of the medical literature. Gynecol Oncol. 2013;130(3):474-482.

5. Piura E, Piura B. Brain metastases from endometrial Carcinoma. ISRN Oncol. 2012;2012:5817493.

6. Lutz S, Berk L, Chang E, et al. Palliative Radiotherapy for bone metastses: an ASTRO evidence-based guideline. Int J Radia Oncol Biol Phys. 2011;79(4):965-976.

7. Foerster R, Habermehl D, Bruckner T, et al. Spinal bone metastases in gynecologic malignancies: a retrospective analysis of stability, protnostic factors and survival. Radiat Oncol. 2014;9:194.

8. Gilbert HA, Kagan AR, Nussbaum H, et al. Evaluation of radiation therapy for bone metastases: pain relief and quality of life. $\mathrm{Am} \mathrm{J}$ Roentgenol. 1977;129(6):1095-1096.

9. Blitzer PH. Reanalysis of the RTOG study of the palliation of symptomatic osseous metástasis. Cancer. 1985;55(7):1458-1462. 